\title{
A rare melanoma feature with primary ovarian origin: a case report and the literature review
}

\author{
Algeri Paola', Rota Sonia Maria², Carlini Laura², Nicoli Elena², Caruso Orlando², Motta Teresio ${ }^{3}$ \\ 1 Department of Obstetrics and Gynecology, University of Milano-Bicocca, MBBM Foundation, San Gerardo Hospital, Monza; Departments of \\ ${ }^{2}$ Obstetrics and Gynecology, ${ }^{3}$ Pathology, Bolognini Hospital, Seriate, Italy
}

Primary ovarian melanoma arising on a mature ovarian cystic teratoma is extremely rare. As best of our knowledge, to date, 49 cases have been reported in literature. Few information was reported about best management and therapy. We present a case occurred in a 69-year-old woman, without symptoms, who come to our unit for stress incontinence. A pelvic mass was detected and, after imaging evaluation, surgery was performed. The diagnosis was ovarian melanoma arose on a mature teratoma. No other adjuvant treatment was proposed after surgery. She died 9 months after the first diagnosis. Primary ovarian melanoma is a definite entity associated with a variable natural history and poor prognosis. Differential diagnosis is a challenge for the pathologist, because it must be differentiated by metastatic melanoma. The corner stone treatment of this disease is surgery; however, chemotherapy, immunotherapy, and target therapy seem to have a role.

Keywords: Ovarian cancer; Melanoma; Teratoma

\section{Introduction}

Mature teratoma or dermoid cystis interested 10\%-20\% of ovarian masses, but malignant transformation occurred in $0.2 \%-2 \%$ of cases. In the major part of cases, the literature reported malignant transformation in squamosus cell carcinoma (88\%), adenocarcinoma, fibrosarcoma, carcinoid tumor, and mixed tumor. Malignant melanoma is an extreme rare kind of transformation [1]. Malignant melanoma has increased dramatically over last decades. Genital tract melanomas usually arise on vulva or vagina. Other gynaecological site are cervical melanoma and ovarian one [2]. Usually ovarian melanomas arise on ovarian teratoma, but there are few cases in which these malignancies are isolated and not associated with an initially benign cyst.

Primary ovarian malignant melanoma was first reported in 1901 [3] and, to date, 49 cases have been described in the world literature, at the best of our knowledge. Table 1 reported a summary concerning the cases presented in literature (complete description of these cases was shown in Supplementary Table 1).

Historically, these cancers have been diagnosed postoperatively because there are no peculiarities symptoms or ultra- sound features. No specific risk factors were described. Its differential diagnosis is a challenge for the pathologist, because it must be differentiated by metastatic melanoma, which is more common. Usually, in case of metastatic cancer the patient reported a history of malignant melanoma and in the ovary, there is not a benign cystic teratoma [4]. At the present, no clear management has been defined for this disease. Generally, the prognosis was poor and the strongest prognostic factors were negative resection margins and stage at diagnosis, but more accurate clinical predictors are required.

Received: 2017.05.27. Revised: 2017.07.07. Accepted: 2017.07.24. Corresponding author: Algeri Paola

Department of Obstetrics and Gynecology, University of MilanoBicocca, MBBM Foundation, San Gerardo Hospital, Via Pergolesi 33, Monza 20900, Italy

E-mail: p.algeri@campus.unimib.it

https://orcid.org/0000-0002-1406-1061

Articles published in Obstet Gynecol Sci are open-access, distributed under the terms of the Creative Commons Attribution Non-Commercial License (http://creativecommons. org/licenses/by-nc/3.0/) which permits unrestricted non-commercial use, distribution, and reproduction in any medium, provided the original work is properly cited.

Copyright (C) 2018 Korean Society of Obstetrics and Gynecology 


\section{Obstetrics \& Gynecology Science}

Algeri Paola, et al. A case of primary ovarian melanoma

Table 1. Summary of published cases of malignant melanoma, according to performed therapy (49 published in literature and our one)

\begin{tabular}{lccc}
\hline Characteristics & Surgical treatment only & $\begin{array}{c}\text { Surgical treatment and } \\
\text { chemotherapy }\end{array}$ & $\begin{array}{c}\text { Surgical treatment, } \\
\text { chemotherapy, and } \\
\text { immunotherapy }\end{array}$ \\
\hline Cases number $\left.(\mathrm{n}=49)^{\mathrm{a}}\right)$ & $31(63.3)^{\mathrm{b})}$ & $10(20.4)^{\mathrm{c}}$ & $7(14.3)$ \\
Mean of patient age at diagnosis $(\mathrm{yr})$ & 49.6 & 46.7 & 46.6 \\
Isolated ovarian melanoma & $3(9.7)$ & 0 & $1(14.3)$ \\
Associated to ovarian sarcoma & $3(9.7)$ & 0 & 0 \\
Associated to ovarian teratoma & $25(80.6)$ & $10(100)$ & $6(85.7)$ \\
Death & $13(41.9) ; 15.20$ mon & $6(60) ; 6.75$ mon & $5(71.4) ; 26.20$ mon \\
Survival & $9(29.0) ; 14.60$ mon & $3(30) ; 39.00$ mon & $2(28.6) ; 6.00$ mon \\
\hline
\end{tabular}

Values are presented as number (\%) and time.

${ }^{a)}$ One case performed no therapy because of the death occurred before; ${ }^{\text {b) }}$ Nine cases lost at follow-up; ${ }^{\text {c }}$ One case lost at follow-up.

\section{Case report}

A 69-year-old woman presented to our unit with a 3 months history of urine stress incontinence. Three years before the patient received a left nephrectomy for a clear cell, grade 2, renal cancer. The oncology follow-up was negative. For over 10 years, she received an annual gynaecological evaluation to check a right ovarian cyst of $3 \mathrm{~cm}$, suggestive for ovarian cystic teratoma. Her medical history included hypertension and hypothyroidism.

At bi-manual pelvic examination, a tender cyst measuring $10 \mathrm{~cm}$ was appreciated, posterior to the uterus. Ultrasound evaluation of the abdomen and the pelvis revealed a right cyst measuring $8 \times 10 \mathrm{~cm}$ compatible with a dermoid cyst. Neoplastic markers were dosed: cancer antigen 125 (CA125) was normal and carbohydrate antigen 19.9 (CA19.9) was elevated at $821 \mathrm{U} / \mathrm{mL}$. Imaging evaluation show a negative chest X-ray; the computed tomography scans confirmed the right adnexal mass, measuring $10 \mathrm{~cm}$, with no ascites; pathologic lymph nodes were not detectable and the liver was normal.

We decide to perform a laparoscopy, which confirmed the imaging described right ovarian cyst. The uterus and left ovary were normal. We done a washing for cytological evaluation. Right oophorectomy was performed with a spilling of sebaceous material and hair. The frozen section revealed carcinoma. So, we converted to a laparotomy to perform a complete surgical staging, used in epithelial ovarian carcinoma, with hysterectomy, contralateral oophorectomy, infra colic omentectomy, multiple peritoneal biopsies and nodes biopsies the end of the surgical procedure, there was no evidence of macroscopic disease. The postoperative course was uneventful.
Histopathological study of the samples revealed mature ovarian cystic teratoma with a solitary, solid and brown-pigmented nodule of $16 \mathrm{~mm}$ which shown pleomorphic spindled cells. The cytoplasm contained abundant brown pigment, confirmed to be melanin. Immunohistochemical evaluation was positive for HMB-45, a specific marker for melanoma, and was also positive for Melan A. Peritoneal fluid, uterus, left adnexal, omentum, peritoneal and nodes biopsies were negative for malignancy.

Microscopic and immunohistochemical images were displayed in Fig. 1.

The patient was re-evaluated and the possibility of a primary melanoma at any other site, mainly skin, eye, and gastrointestinal tract was ruled out; so primary ovarian melanoma was confirmed. After being reviewed by a medical oncologist specialising in melanoma care, the patient was advised that adjuvant therapy would not be necessary. A follow-up was arranged at our outpatient clinic. We observed a normalization of CA19.9 within 3 weeks. Seventeen days after the first surgery the patient was hospitalized with abdominal pain. The clinical evaluation, abdominal radiography and abdominal ultrasound did not show any evidence of disease. The clinical follow-up, performed 3 months after surgery, did not show any signs of illness. The markers were fully normalized. Additional 4 months after, the patient came to our emergency service presenting abdominal pain. Clinical examination showed the presence of a solid pelvic mass of $4.3 \times 3.3 \mathrm{~cm}$. CA125 was $26 \mathrm{U} / \mathrm{mL}$ and CA19.9 was $3 \mathrm{U} / \mathrm{mL}$. The tomography scan confirmed the pelvic mass and detected multiple metastatic lesions in the lung parenchyma of a variable diameter between 0.7 and $1.2 \mathrm{~cm}$. Multiple liver metastases measuring 


\title{
Obstetrics \& Gynecology Science
}

\author{
Vol. 61, No. 2, 2018
}
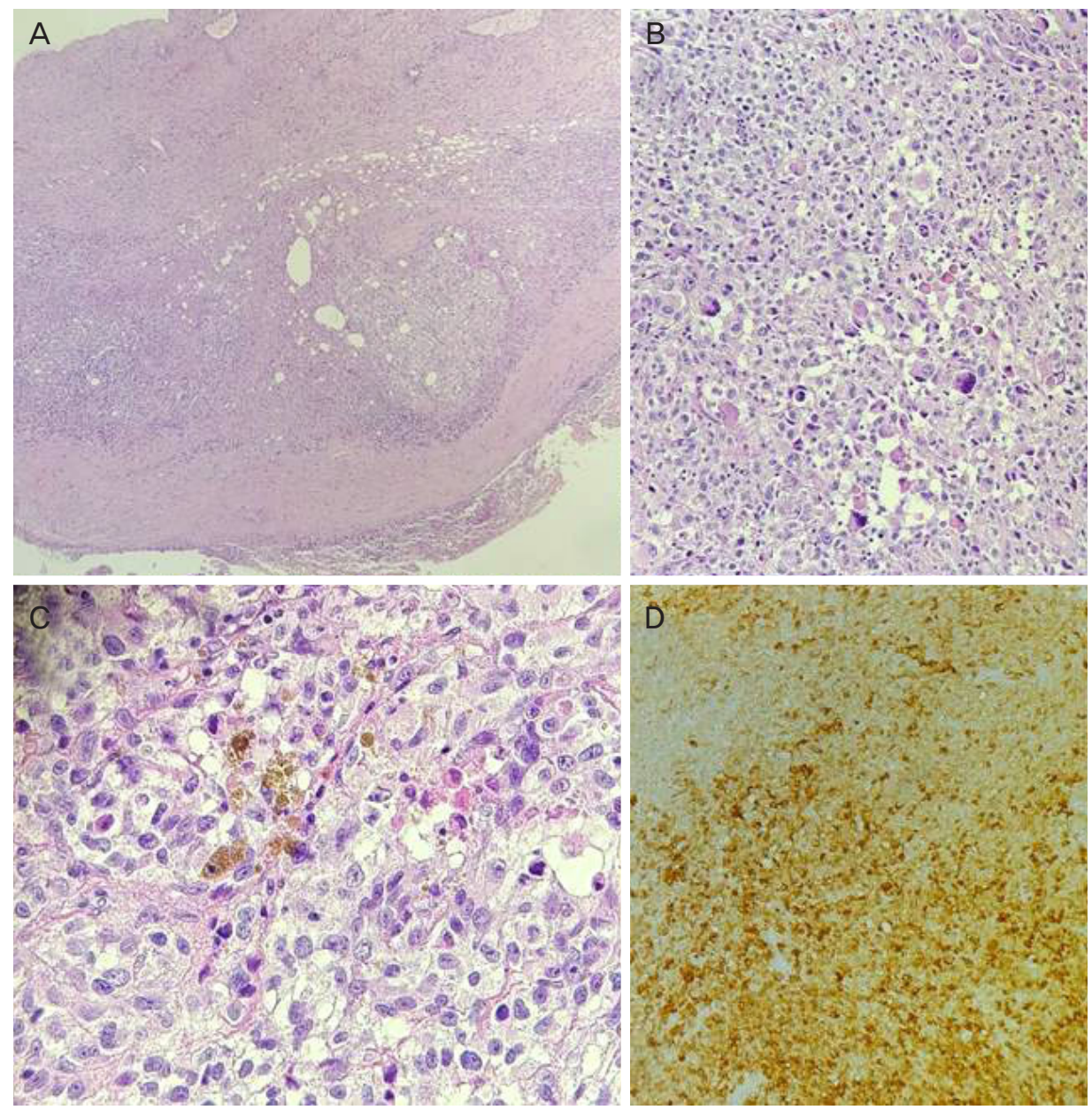

Fig. 1. (A) cyst wall (original magnification $\times 10)$. (B) hematoxylin-eosin (original magnification $\times 40$ ). (C) hematoxylin-eosin with brown pigment (original magnification $\times 40$ ). (D) Immunostain for Melan A shows a diffuse, intensively cytoplasmic positive reaction.

in diameter from 6.5 to $1.9 \mathrm{~cm}$ were also found. There were multiple enlarged lymph nodes in the iliac and para-aortic sites and multiple metastases of varying diameters of 1.8 and $2.6 \mathrm{~cm}$ on the abdominal wall. The patient presented quick progression with bowel obstruction and rapid decline of perform status. She refused chemotherapy and died 2 months later (9 months after the first surgery).

\section{Discussion}

Malignant melanomas involving the ovary are more frequently metastatic than primary in nature [4]. Primary melanomas of the ovary are thought to arise on malignant transformation of a mature teratoma, which occurred in only $0.2 \%-2 \%$ [1]. Of these, the most common are squamous cell carcinomas. Less commonly, the diagnosis is adenocarcinoma, fibrosarcoma, carcinoid tumor or mixed tumors, in order of decreasing fre- quency. Malignant melanoma is the rarest tumor arising on a benign cystic teratoma of the ovary and the possibility of a secondary involvement of the ovary form a tumor arising on another place must always be excluded [4]. Ovarian melanoma could also be isolated or associated to other kind of ovarian cancer, as described in few cases reported in literature.

Our case is a primary malignant melanoma of the ovary. In fact, it meets the criteria laid down by Cronje and Woodruff [5] and confirmed by Boughton et al. [6]. Specifically, the absence of primary extra-ovarian tumor, an unilateral ovarian tumor with associated teratoid element, the demonstration of melanocyte junctional activity (not mandatory for diagnosis) and the good correlation of the patient's age and symptoms with those of other cases well documented in the literature. Even if it is not pathognomonic, an increase of CA19.9 was described in literature, especially if the melanoma arose on a mature teratoma, as reported in our case [7]. Primary ovarian melanoma was first described by Andrewes in 1901 [3]. Since 


\section{Obstetrics \& Gynecology Science}

then, 49 cases have been reported in the world literature, summarized in Table 1 (Supplementary Table 1, reported the complete description of these cases).

Primary malignant melanoma arising on the ovary does not have a good outcome, even in the early presentation confined to one ovary. Patient age ranges from 19 to 86 years (median 49.1). The presenting symptoms are due predominantly to an enlarged ovary causing abdominal distension and pain. All cases in the literature, with one exception, underwent laparotomy with surgery ranging from an ovarian cystectomy to extensive debulking. Two patients received surgery during pregnancy. Sixteen patients received chemotherapy and 7 cases immunotherapy. In one case, radiotherapy was used with a year of disease free survival. In addition, target therapy was used in one case, with long (69 months) follow-up without evidence of disease. High mortality rate is described: 23 $(46.9 \%)$ of the 49 cases published, died due to the disease within 1 days and 18 months from first diagnosis. Follow-up was not available for 10 patients (21.7\%). One patient was lost to follow-up after 6 months, one after 1 year. A longterm follow-up is available for only 2 patients reported by Carlson and Berger (Supplementary Table 1), who demonstrated a longer than 5 years survival, with no evidence of disease. The follow-up survival rate for other patients is shorter and has a range between 1 and 30 months.

In summary, primary malignant melanoma of the ovary was an extremely rare and definite entity, associated with a variable natural history and poor prognosis; compared to ovarian carcinoma they reported a worst prognosis [7]. The pattern of spread is to adjacent structure and to lymphatic and haematogenous routes (lung, liver, and bone) [7]. The optimum treatment for this cancer remains to be defined, but review of the literature suggests a primary role for surgical resection of all tumor [8].

Chemotherapy (with different regimens consisting of dacarbazine, cisplatin, and taxol) and immunotherapy could be considered as adjuvant treatment and recent study reports a role of target therapy [9]. Despite treatments used, patient outcome was unpredictable.

\section{Conflict of interest}

No potential conflict of interest relevant to this article was reported.

\section{Supplementary materials}

Supplementary table associated with this article can be found online at https://doi.org/10.5468/ogs.2018.61.2.282.

\section{References}

1. O'Gorman T, Olaitan A. Primary malignant melanoma arising in an ovarian cystic teratoma. Eur J Gynaecol Oncol 2009;30:88-9.

2. Sugiyama VE, Chan JK, Kapp DS. Management of melanomas of the female genital tract. Curr Opin Oncol 2008;20:565-9.

3. Andrews HR. Primary Melanotic sarcoma of the ovary. Trans Obstet Soc (Lond) 1901;43:228-31.

4. Moore RG, Chung M, Granai CO, Gajewski W, Steinhoff $\mathrm{MM}$. Incidence of metastasis to the ovaries from nongenital tract primary tumors. Gynecol Oncol 2004;93:87-91.

5. Cronje HS, Woodruff JD. Primary ovarian malignant melanoma arising in cystic teratoma. Gynecol Oncol 1981;12:379-83.

6. Boughton RS, Hughmanick S, Marin-Padilla M. Malignant melanoma arising in an ovarian cystic teratoma in pregnancy. J Am Acad Dermatol 1987;17:871-5.

7. Hyun HS, Mun ST. Primary malignant melanoma arising in a cystic teratoma. Obstet Gynecol Sci 2013;56:201-4.

8. Vimla N, Kumar L, Thulkar S, Bal S, Dawar R. Primary malignant melanoma in ovarian cystic teratoma. Gynecol Oncol 2001;82:380-3.

9. Puzanov I, Flaherty KT. Targeted molecular therapy in melanoma. Semin Cutan Med Surg 2010;29:196-201. 